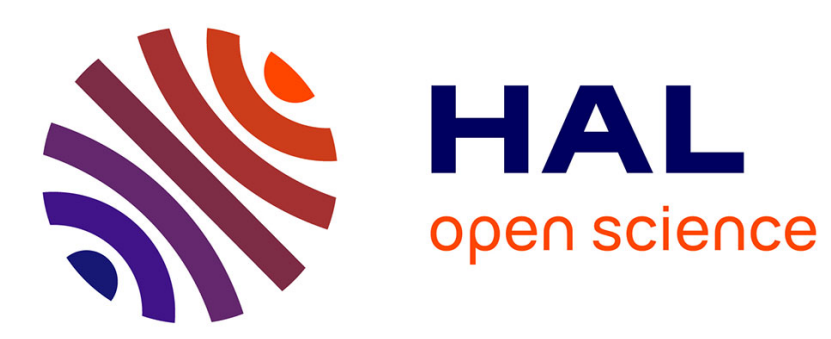

\title{
Le rapport au savoir de deux jeunes scolarisés en ITES : l'impossible intériorité et la mise au défi
}

\author{
Françoise Hatchuel
}

\section{To cite this version:}

Françoise Hatchuel. Le rapport au savoir de deux jeunes scolarisés en ITES : l'impossible intériorité et

la mise au défi. Cahiers de psychologie clinique , 2009, 33 (2), 10.3917/cpc.033.0121 . hal-01658256

\section{HAL Id: hal-01658256 \\ https://hal.parisnanterre.fr/hal-01658256}

Submitted on 7 Dec 2017

HAL is a multi-disciplinary open access archive for the deposit and dissemination of scientific research documents, whether they are published or not. The documents may come from teaching and research institutions in France or abroad, or from public or private research centers.
L'archive ouverte pluridisciplinaire HAL, est destinée au dépôt et à la diffusion de documents scientifiques de niveau recherche, publiés ou non, émanant des établissements d'enseignement et de recherche français ou étrangers, des laboratoires publics ou privés. 


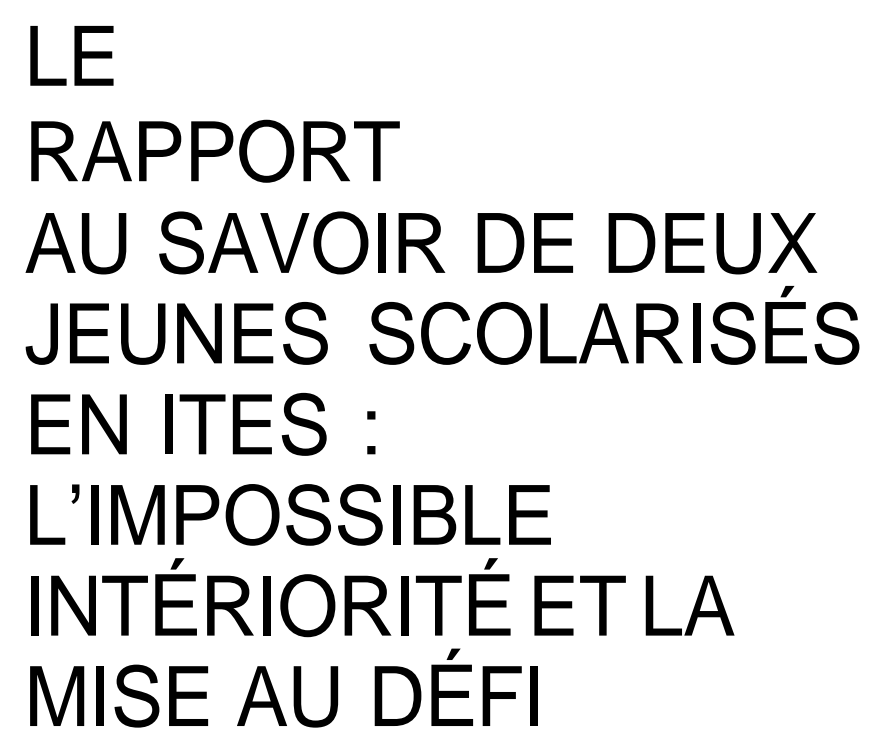

Françoise

HATCHUEL 


\section{Le rapport au savoir de deux jeunes scolarisés en ITES : l'impossible intériorité et la mise au défi. Introduction}

La question des effractions de et à l'adolescence sera étudiée ici du point de vue des effractions psychiques, en s'appuyant sur une recherche questionnant le rapport au savoir de jeunes garçons de 12 à 16 ans scolarisés dans un institut géré par l'Association de Sauvegarde de l'Enfance et de l'Adolescence des Yvelines (voir Hatchuel, Hans, Clerc, Carlin, 2005). Conformément à l'orientation de notre équipe de recherche, nous avons travaillé dans une perspective clinique telle que la définit Claude Revault d'Allones (1989), en faisant résonner en nous-mêmes nos propres mouvements transférentiels vis-à-vis des données de recherche (voir aussi Devereux 1967-1980) et en nous situant en sciences de l'éducation, c'est-à-dire en interrogeant prioritairement non pas des cas, mais des situations (éducatives notamment) et la façon dont certaines sont susceptibles de réactualiser ou de contenir certains conflits psychiques $^{1}$.

L'association commanditaire de la recherche souhaitait non seulement mieux comprendre ces adolescents qui lui étaient confiés, mais aussi tenter d'enrôler l'équipe éducative dans une refondation du projet pédagogique de l'établissement. La négociation qui s'en est suivie nous a amené(e)s à délier ces deux objectifs et à nous centrer sur les adolescents, sur la base d'entretiens non-directifs enregistrés, menés avec une douzaine d'entre eux à partir d'une consigne portant sur l'apprentissage (« peux-tu me dire, très librement, comme ça te vient,

\footnotetext{
${ }^{1}$ Nous avons tenté de cerner (voir Blanchard-Laville, Chaussecourte, Hatchuel, Pechberty, 2005), les questions méthodologiques, épistémologiques et éthiques posées par une telle démarche en sciences de l'éducation, ainsi que les grandes thématiques qui s'en dégageaient, tandis que j'ai présenté (voir Hatchuel 2005-2007) les principaux résultats obtenus au sein de notre équipe sur l'approche psychanalytique du rapport au savoir.
} 
comment ça se passe pour toi quand tu apprends ? »). Mais cette volonté initiale de l'association de tutelle provoquera une méfiance forte de la part d'une partie de l'équipe éducative. Cette méfiance sera notamment perceptible lors de notre première visite à l'Institut, visite au cours de laquelle nous avons rencontré collectivement les jeunes et les adultes, et où plusieurs éducateurs nous ont fait ressentir leur méfiance quant à l'utilité du travail que nous allions entreprendre. Ce sera un élément important dans l'analyse des entretiens.

Tout l'enjeu de mon propos va en effet consister à montrer comment les effractions psychiques circulent en permanence dans l'institution, selon le principe d'homologie fonctionnelle tel que l'ont décrit Jean-Pierre Pinel et Sylvie Morel (2001), où les psychismes des uns et des autres se contaminent mutuellement via certains éléments (ici le fantasme d'intrusion). En lien avec la notion que je tente de théoriser de «fiction de soi » (voir Hatchuel 2009), c'est-à-dire cette histoire que chacun(e) de nous tente de se raconter à partir de ce qui lui est dit de lui ou d'elle-même, je montrerai notamment comment cette circulation des effractions impacte ces fictions de soi pour les rendre « insoutenables » via une mise au défi où le psychisme effracté ne peut exister que dans l'extériorisation du passage à l'acte. L'analyse de deux entretiens, dont l'un quasi-muet, avec deux jeunes prénommés Mathieu et Stéphane montrera, à travers mes propres mouvements transférentiels, comment peut se recréer un espace d'intimité partagé qui redonne à chacun sa propre intimité.

\section{Contexte de la recherche et lien aux interviewés}

La question de mon lien à ces deux adolescents au moment où je les interviewe renvoie à ma propre posture de responsable de la recherche, qui m'engage face aux différents groupes d'adultes partenaires : association commanditaire ; équipe pédagogique de l'institut ; équipe de recherche au sein de laquelle j'évolue, passant progressivement du statut d'étudiante à celui de chercheuse confirmée ; et enfin, groupe constitué sous ma responsabilité pour répondre à la commande telle qu'elle a été finalement négociée et qui se compose de deux 
collègues proches qui acteront par ce travail leur entrée officielle dans notre équipe de recherche, deux étudiantes dont l'une continue à travailler aujourd'hui sous ma direction et un formateur d'éducateurs.

C'est ce groupe qui est chargé de soutenir la fiction d'une recherche possible et de ma capacité à la diriger, et c'est à ce moment-là que le terme à émergé pour souligner l'interaction permanente de la réalité et des imaginaires La fiction «soutenable » est alors celle qui sera à la fois suffisamment proche du désir pour constituer un moteur et suffisamment « réaliste » pour que l'on puisse raisonnablement espérer s'en approcher, dans un processus toujours incertain de construction d'une certaine forme de croyance en soi étayée par le regard d'autrui, entre intériorité psychique et appropriation d'éléments extérieurs. Et c'est ce processus que j'ai étendu ensuite à la construction de soi des adolescents, après avoir commencé à le percevoir dans la constitution du groupe de recherche.

Lorsque nous arrivons pour mener les entretiens (il a été convenu que nous venions par deux, nous installant chacun(e) dans une salle où viendraient nous rejoindre les jeunes volontaires, accompagnés par leur éducateur), je me sens « responsable de la recherche » et $\mathrm{j}$ ' ai envie de prouver que nous allons pouvoir faire du bon travail. Je suis aussi sensible aux différents enjeux des uns et des autres et, déjà, aux circulations que nous avons pu repérer : difficulté à protéger l'intimité des jeunes (il nous sera proposé de visiter une chambre, ce que nous refuserons), les espaces de rencontre (les premiers entretiens effectués par d'autres membres du groupe ont été l'occasion de tentative d'intrusion de la part de jeunes se considérant comme «laissés à la porte», au point qu'il sera proposé à l'une de nous de «[s]'enfermer pour être tranquille ») ou l'institut lui-même, dont le caractère d'espace non clos est mis en question. Nous avions également noté, lors du passage dans un cours de mathématique, l'attitude ironique de l'enseignant s'adressant à un jeune pour souligner qu'il faisait «moins le fier que tout à l'heure maintenant qu'il y a des personnes extérieures ». 


\section{Mathieu ou l'impossible intériorité}

\section{Le premier moment : la captation de la dette}

Mathieu est le premier jeune que je rencontre. Calme, poli, réservé, il cultive une apparence de «petit garçon » «idéal ». Le chef de service qui l'accompagne me le présente comme particulièrement méritant puisqu'il accepte, dit-il, de coopérer à la recherche alors qu'il vient juste d'arriver. L'élaboration de la gêne provoquée par cette phrase me conduit à son propos à parler de «captation de la dette » : dans la mesure où rien, ni dans l'attitude de Mathieu, ni dans celle de l'adulte, ni dans mon ressenti, ne me pousse à accorder à Mathieu lui-même le bénéfice de son "mérite », et où je me sens pourtant bien en dette, il m'apparaît que cette dette semble créée par l'adulte pour son bénéfice propre, avec l'accord inconscient de Mathieu, sans doute habitué à être mis dans cette position. Effraction, donc, de ce qui appartiendrait en propre à Mathieu, ce qui lui est dû. Une première piste d'analyse peut ainsi être de considérer que beaucoup de jeunes dits «en difficulté » le sont avant tout parce que, n'ayant pas appris à délimiter un espace personnel cohérent, ils peuvent ainsi être mis au service des projets inconscients d'autrui, le processus risquant d'être renforcé lorsque les adultes qui les accompagnent s'en saisissent inconsciemment pour obtenir de la reconnaissance en échange de leur « dévouement ».

Tonalité générale : l'impossible engagement

En cohérence avec cette difficulté à exister comme sujet, Mathieu refuse tout autre échange que celui d'une « questionréponse » mécanique auquel j'ai beaucoup de mal à échapper. Tout son être semble tendu vers le besoin de répondre «ce qu'il faut » et, finalement, de n'avoir rien à dire en propre. Je tourne donc un peu désespérément en rond à la recherche de «la » bonne question, en miroir de lui qui cherche «la» bonne réponse. L'expression «C'est tout ». reviendra 19 fois au cours de l'entretien, soit en moyenne toutes les minutes et demie. Cette utilisation d'expressions « clôturantes » est assez fréquente chez les adolescents, comme en témoignent plusieurs de nos travaux précédemment cités. Elle est donc à 
analyser à la fois en tant que telle, comme besoin de se rassurer sur le fait qu'on ne sera pas entraîné plus loin qu'on ne le souhaite, mais aussi sur le contenu qui semble ici bien avoir sa valeur propre. Lorsque Mathieu précise «Y a rien d'autre » ou «J'ai rien à dire » (10 utilisations du terme «rien»), il semble me signifier l'incompréhensibilité de mon acharnement : que puis-je bien vouloir s'il n'y a rien à dire ?

Le tout est dit avec une diction extrêmement soignée, notamment lorsque je manifeste des signes de doute ou d'incompréhension : situations où Mathieu n'essaye pas d'expliquer ou de développer, mais reprend strictement ce qu'il venait de dire, le plus posément possible, en détachant soigneusement les syllabes. Comme s'il fallait que tout soit immédiatement compréhensible, clair, lisse, transparent, et qu'il n'existait aucune place pour l'équivoque ou l'ambiguïté. J'ai presque l'impression, en le voyant s'appliquer ainsi à répéter, d'entendre un «bon sang mais articule quand tu parles! » qui lui aurait été asséné régulièrement. Cela me renvoie, a contrario, au débit ultra rapide, au langage particulier et aux signes codés des «tribus » adolescentes qui construisent ainsi leur espace d'autonomie à l'abri du regard des adultes.

\section{Apprendre, travailler : des termes sans épaisseur}

En fait, ce qui caractérise Mathieu, c'est une tentative permanente de coller absolument à son propre langage, donnant aux mots un sens strictement descriptif, sans aucune allusion aux processus internes auxquels ils pourraient correspondre. Face aux difficultés d'apprentissage, d'abord déniées puis évoquées comme passagères, Mathieu répond que, dans ces caslà, il se dit qu'il «réapprendr[a] / un autr'jour », confondant, comme le fait d'ailleurs la langue, l'action et le résultat, puisqu'il semble davantage signifier « je me replacerai ultérieurement en situation d'essayer d'apprendre » que «j'acquerrai un nouveau savoir ». On peut ainsi apprendre indéfiniment mais ne jamais savoir. Et ce d'autant plus que le savoir, visiteur décidément mystérieux, peut venir et repartir : « Non des fois $\mathrm{j}$ 'apprends mais j'oublie vite». Cette confusion entre savoir et comportement apparent, que l'on peut relier à une impossibilité d'accéder à son intériorité, se retrouvera à plusieurs reprises dans l'entretien, comme le montre également 
l'emploi du terme «travail », terme apparemment magique et passe-partout. La première fois qu'il l'emploie, c'est quand je lui demande d'évoquer sa journée (1. 154) : « Ben aujourd'hui j'ai travaillé ». Lorsque je le relance sur ce point, il précise (l. 160) : «Ben oui j'ai eu des d'voirs. Des contrôles/// C'est tout ». Les mots semblent, là encore, vides de sens, comme s'il s'agissait pour lui avant tout de faire comme les autres, et probablement comme les adultes. On peut alors faire l'hypothèse qu'il ne peut pas se situer autrement que par des désignations qui lui sont extérieures.

\section{Une subjectivité extrêmement ténue}

Les choses se dénoueront un peu lorsque je le relance, après un long silence, sur l'ensemble des « choses-là dont on... vient d'parler» (1. 197) et qu'il revient de lui-même sur ce qu'il aime ou non, s'autorisant ainsi pour la première fois à ne pas répondre directement à une question posée. Il aura fallu pour cela une vingtaine de minutes d'entretien au cours desquelles, si je n'ai pas réussi à ce que Mathieu sorte de son désir de bien faire, je serais au moins parvenue à tenir une posture d'écoute sans tomber dans sa demande de juger de la crédibilité de ses propos ou de la valeur de ses actes. C'est-à-dire que je lui ai laissé l'entière responsabilité d'estimer, et donc de questionner, l'écart entre ses propos et la réalité, tout en lui renvoyant bien que le résultat de ce travail m'intéressait. Ce faisant, il peut donc élaborer quelque peu autour de ce qui semble être sa problématique centrale, à savoir se demander ce qu'il a le droit de dire ou de faire, et selon quels critères, et laisser ainsi émerger quelque chose de sa subjectivité. Mais c'est comme en apnée (ce qui me fait penser que se joue-là une tentative de dénouer quelque peu le conflit sans que Mathieu soit totalement certain de pouvoir y parvenir) qu'il se lance dans une réponse où il s'anime enfin un peu et où il peut supporter de confronter deux assertions contradictoires (on peut noter à cet égard c'est la première fois qu'il emploie la conjonction «mais ») :

$\mathrm{M}$ : Ben les devoirs par exemple j'aime ça

$\mathrm{FH}: \mathrm{Mm} \mathrm{mm}$

M : J'aime travailler mais tout c'qui est jardin, les tables, la vaisselle/non. 
Il faut souligner que, contrairement à ce que le saut de paragraphe semble indiquer, les propos de Mathieu ne s'interrompent pas, ils sont seulement suspendus, et je ressens cette suspension comme un besoin impératif d'être autorisé à continuer, autorisation que je m'empresse de donner. On peut imaginer que c'est ce qui l'aide à pouvoir soutenir dans le même élan d'une part les mots représentatifs d'une image de lui qui serait de l'ordre de la survie (un jeune n'aimant pas les devoirs ne saurait avoir le droit d'exister), d'autre part quelque chose de sa réalité interne : quand il s'agit concrètement de travailler, finalement, non, il n'aime pas. Ceci étant, il a probablement atteint là une limite et s'empresse de m'empêcher de lui en demander plus : «Et puis c'est tout y a rien ».

\section{L'inquiétante rencontre}

À ce niveau de l'analyse, je mesure donc la difficulté pour Mathieu à exister par lui-même, et à faire exister les autres de façon autonome (difficulté à nommer les personnes, identification «à l'identique » à son père, dont il évoque qu'il le « remplace » lorsqu'il se blesse mais sans rien dire de ce que recouvre ce «remplacement», etc.). Je me sens tellement peu autorisée à entrer en contact avec lui que je me surprends, à plusieurs reprises, à m'effacer moi-même en posant des questions dont la réponse ne s'adresserait pas à moi : «Tu peux raconter ça un peu comment ça s'est passé ? » (et non pas «tu peux me raconter », (1.63)). Ce que me demande finalement Mathieu, et à quoi je résiste très difficilement, c'est d'être présente par mes questions, de lui donner quelque chose à faire, mais sans pour autant me poser comme sujet, de la même façon que lui-même ne peut se poser comme être humain autonome et soutenir une quelconque fiction de lui-même.

Je ressens alors cette fermeté à n'avoir rien à dire comme une défense extrêmement solide visant à prévenir toute intrusion de ma part dans son espace psychique qui semble ne pouvoir être préservé qu'au prix de l'absence d'interaction. L'accent mis sur le terme «travail » pourrait alors être interprété comme une tentative d'échapper à l'emprise : dans un milieu où le travail semble considéré comme une valeur positive (son père aménage lui-même la maison), dire " j'ai travaillé » pourrait alors être un moyen de satisfaire les adul- 
tes et d'éviter qu'ils n'en demandent davantage, provoquant ainsi un début de séparation et de protection de son intimité. En termes psychanalytiques, on pourrait alors dire que la valeur « travail », incarnation du surmoi freudien et de la prise en compte du réel, parviendrait bien ici mettre en échec le moi-idéal, toujours à la recherche d'une fusion régressive. Reste qu'ici le travail n'est qu'un mot, non une pratique réelle et engagée, si bien que ce début de séparation ne peut aboutir pour Mathieu à une quelconque réalité.

\section{Une fiction de compréhension totale}

Pris son exigence de clarté et de transparence, Mathieu semble ne pouvoir s'autoriser à avoir des doutes et des difficultés. Je fais l'hypothèse qu'une relation fusionnelle à sa mère construit la fiction (malheureusement non soutenable) d'une compréhension totale et immédiate conduisant Mathieu à une impossibilité de se définir par lui-même qui risquerait de le faire sortir de ce qu'autrui pense pour lui. Interdit de conflictualité, il ne peut construire une intériorité qui lui appartiendrait en propre, ce qui l'empêche de prendre des risques et de se prononcer. Tout se passe comme si, le conflit s'avérant trop angoissant pour son entourage (et probablement en premier lieu sa mère), il avait été mis à une place où il se devait d'être l'incarnation du non-conflit. On rejoindrait alors ce que C. Geffray (2001) appelle le « refoulement du doute », où un individu est chargé par les autres d'incarner une réponse aux angoisses. Mais là où $\mathrm{C}$. Geffray étudie comment, par exemple, le chef d'une tribu amérindienne se doit d'incarner celui qui n'a pas peur de la mort, aidé en cela par son statut, même si cela lui impose de douloureuses obligations, c'est ici un enfant qui aurait la lourde tâche de faire barrage aux angoisses des adultes, position évidemment intenable pour Mathieu. Il ne peut alors rien construire de lui-même, et il est probable qu'une des premières urgences pour lui serait de travailler le conflit et la séparation, et de percevoir que ni l'un ni l'autre ne sont destructeurs.

Or, en écho à une réflexion du groupe qui s'est dit que les interruptions durant les entretiens étaient un analyseur, et qu'elles montraient, probablement, la nécessité de travailler les séparations et les sorties, il me semble, à l'analyse de l'entretien 
de Mathieu, que probablement les entrées peuvent aussi être un espace d'interrogation à prendre comme tel. Comment intégrer un groupe sans s'y perdre ? C'est bien la porosité de l'institution qui est en jeu, sa capacité respirante d'échange avec l'extérieur et donc son degré de vie. Or, Mathieu me semble très vite capté, avalé, digéré par l'institution, il se fond immédiatement dans le miroir qu'on lui offre et ne peut donc, pour l'instant, s'y construire.

\section{Stéphane : du défi à la rencontre}

\section{Tonalité générale et premiers moments}

L'entretien mené avec Stéphane est tout à fait particulier dans la mesure où, dès la première minute, celui-ci s'installe dans un mutisme dont je ne parviendrai pas à le faire sortir malgré de consciencieuses relances «techniques ». Après Mathieu le bon élève sage, c'est moi qui me met à jouer la bonne élève, face à Stéphane qui prend le rôle de l'élève réfractaire. Je me sens prise entre mon désir de respecter la parole (ou la nonparole) de Stéphane, et l'inquiétude d'une chercheuse qui se veut spécialiste du recueil de la parole des adolescent(es) et voit bien qu'elle a atteint ici ses limites. Je me raccroche donc à ce que je sais faire (l'écoute et les relances « accompagnantes ») pour tenter de «limiter les dégâts », pour Stéphane comme pour moi.

J'éprouve, dans le même temps, une relative sérénité quant à la conscience que j'ai d'être en difficulté. Il me semble que j'ai le droit de ne pas savoir faire, que c'est une des conditions de la recherche que de prendre ce risque. L'image qui me vient alors est celle d'un oral de concours d'école d'ingénieur ${ }^{2}$ où l'examinateur, pour me piéger, m'a donné un exercice très proche d'une formule hors programme mais couramment enseignée. Après un premier mouvement de panique je me suis dit que j'avais le droit de ne pas la savoir, que de toutes façons je ne la savais pas et qu'il était inutile que je perde du temps à chercher, et que j'allais donc faire comme j'avais l'habitude : regarder sur des exemples et utiliser les points de programme sur lesquels j'étais plus assurée. 
Nous sommes donc ici en plein paradoxe clinique : nous savons faire et pourtant ce que nous savons faire ne servira pas, ou en tout cas pas directement. Nos connaissances et notre technique servent à mettre en place une routine qui nous laisse un peu plus d'esprit disponible pour tenter de ressentir quelque chose de la situation. En l'occurrence, je crois que très vite, je vis ce moment comme un jeu, une sorte de défi, mais plutôt ludique, qui m'est posé à la fois par Stéphane et par le directeur adjoint qui l'a accompagné.

C'est en effet le chef de service pédagogique qui vient chercher Mathieu, et il est accompagné de Stéphane, alors que la question des transitions entre deux entretiens nous avait été précédemment posée et que nous avions signifié notre souhait de disposer de quelques minutes entre chaque. Je suis donc placée d'emblée devant un fait accompli et une injonction à accepter d'autres règles que celles qui avaient été fixées, et je le ressens, à mon tour ai-je envie de dire, comme un envahissement de mon espace personnel. Le terme utilisé pour me demander si j'accepte de recevoir Stéphane («alors, on enchaîne ?») et le ton de défi utilisé me mettent mal à l'aise mais, dans la mesure où cet enchaînement est présenté comme la conséquence logique de la question précédente (est-ce que cela s'était bien passé avec Mathieu), je suis piégée : ne pas recevoir Stéphane immédiatement me rendrait suspecte d'incompétence. Nous sommes là exactement dans ce que j'identifierai plus tard comme la logique de défi où c'est l'extérieur, et non le sujet lui-même, qui définit les épreuves (de préférences impossibles), chargées de soutenir la fiction de soi. Je relève donc le défi et accepte cet « enchaînement » («Puisqu'il est là, oui, je vais le recevoir »).

Le défi avait d'ailleurs commencé à s'enclencher plus tôt, puisqu'à cette question de savoir si tout s'était bien passé à laquelle Mathieu m'avait laissée répondre, le ton déjà quelque peu narquois de cet adulte «m'attendant au tournant » m'avait incitée à répondre que oui et que je ne mangeais personne. Mais ce faisant, sous couvert d'humour, je déplaçai l'interrogation du terrain de la recherche, que je cherchais à protéger, à celui de la rencontre; et ce d'autant plus que, probablement culpabilisée par ce que je ressentais de la souffrance de Mathieu, j'avais là l'occasion de lui signifier que, si j'avais 
provoqué cette souffrance, du moins n'était-ce pas volontaire. Mais, ce faisant, le but de l'interaction devenait non plus d'écouter la parole de Mathieu mais de le préserver de toute agression potentielle émanant d'une femme, ce qui semble être une de ses angoisses propres : en tentant déjouer le défi de l'adulte, j'adhérais aux peurs de l'adolescent, dans un mécanisme probablement relativement banal, où nous voyons comment la force des enjeux des jeunes, alliés à nos propres enjeux d'adultes, nous empêchent de tenir le cadre d'une rencontre qui se placerait dans un registre socio-professionnel ${ }^{3}$, me conduisant, qui plus est, à mettre sur la place publique le peu d'intimité que j'avais réussi à instaurer entre Mathieu et moi.

\section{Le défi de Stéphane}

Je suis donc inquiète, peu satisfaite de l'entretien avec Mathieu, n'ayant pas eu le temps d'y réfléchir, et pas en condition d'accueillir Stéphane (le récit détaillé des péripéties précédentes montre évidemment la difficulté à réunir ces conditions). Je me retrouve face à Stéphane qui, lui, semble être venu en traînant les pieds, sans doute sollicité parce qu'il était sur le chemin de l'adulte, lui évitant ainsi d'avoir à faire un trajet supplémentaire. J'exprime donc ma consigne d'un ton appliqué où l'inquiétude perce probablement (que vais-je donc pouvoir faire de ce jeune réfractaire après la surface lisse présentée par le précédent ?). La réponse fuse immédiatement : après une demande de précision («Quand j'apprends quoi ? »), entraînant un «n'importe » quasi catégorique de ma part, Stéphane semble plus que perplexe sur la pertinence de ma question : "Ouais mais ça m'aide pas là vot'question aussi ». Je relance alors avec un parfait modèle de non-directivité : «C'que tu aimes/c'que tu aimes pas / c'qui... » et là, la réponse est encore plus immédiate : « Ici ? ». Il veut parler de son expérience à l'institut, et la force de ce désir, qui semble confiner à la révolte, me désarçonne, si bien que je ne parviens pas à m'en emparer. Je tente donc de concilier mon désir et le sien (« Dans l'idée d'apprendre// mais ça peut être ici oui ») mais le compromis semble impossible pour Stéphane, qui secoue la tête opiniâtrement et répond qu'il ne « voi[t] rien à dire/ sur ça » quand je relève ce mouvement de tête. À partir

${ }^{3}$ Pour reprendre la théorisation de Gérard Mendel (1993) qui distingue les registres socioprofessionnels et psychoaffectifs, en montrant comment nous confondons très facilement les deux. 
de là, le dialogue s'interrompt. Stéphane précisera que cette situation lui semble extrêmement suspecte ( «Ch'sais pas moi j'demande plus de détails avant d'vous faire confiance/c'est tout ») et je ne saurai pas créer cette confiance qu'il demande. Je le relance sur sa demande de détails, alors qu'évidemment elle masque une autre demande, implicite, que l'on pourrait résumer en une sorte d'injonction paradoxale : «débrouillezvous pour me mettre en confiance mais je ne vous donnerai rien qui puisse vous indiquer ce qui pourrait me rassurer ». Comme si Stéphane me mettait au défi, là encore, de savoir qui il est (je n'emploie évidemment pas ici le terme de savoir de façon anodine) sans me donner les outils pour cela. Faudrait-il que je sache sans avoir appris ? Son rapport au savoir sous-entend-il cela, qu'il ne peut pas me répondre, puisque l'apprentissage n'est pas un processus conscient pour lui ?

Sur ce point, nous nous étions dit, en discussion d'équipe, que la situation d'entretien faisait largement écho à notre questionnement sur le savoir, dans la mesure où les jeunes ne savent pas ce que nous savons d'eux, de même que nous ne sommes pas très assurées de ce qu'ils savent de nous. Le défi, modalité particulière d'interaction relativement courante entre les jeunes pourrait alors être une modalité de réponse à ce type de questionnement. Les travaux de Pierre Merle (2005) sur l'humiliation subie par les élèves nous incitent d'ailleurs à penser que cette modalité de réponse est probablement contaminante pour les institutions éducatives. Relever un défi c'est, en effet, accepter de défendre bec et ongle une fiction de nousmêmes qui nous est donnée de l'extérieur, alors qu'une conception de la fiction comme transitionnalité demanderait, au contraire, que nous puissions « jouer» (au sens de Winnicott) avec cette fiction afin de trouver la juste distance de nousmêmes que nous pouvons soutenir. C'est ce travail d'élaboration entre soi et le monde extérieur qui ne peut se faire s'il nous faut en permanence mettre en scène ce que nous sommes et en justifier devant autrui. Il me semble que lorsque j'ai évoqué un peu plus haut comment j'avais, pour ma part, pris le défi comme un jeu, c'est probablement parce que le travail clinique, accompagné de la protection que me confèrent statut social et expérience, pouvaient me permettre, justement, de réintégrer ce défi dans mon espace intérieur et de m'affranchir dans une certaine mesure des mises au défi extérieures. Ce 
n'est, à cet égard, probablement pas pour rien que la situation qui m'est revenue est celle d'un concours d'entrée dans une grande école, que l'on peut considérer comme un rituel de passage relativement efficace, dans le sens où il s'agit d'épreuves difficiles et compétitives, mais qui garantissent à l'arrivée une réussite relative, puisqu'un(e) élève de classe préparatoire est à peu près assuré(e), au bout du compte, d'intégrer une école, quelle qu'elle soit, qui lui conférera un statut social « raisonnable » et n'a donc pas à défendre son droit fondamental à devenir un(e) adulte à peu près intégré(e) dans la société. Les jeunes de l'ITES sont probablement à l'exact opposé de cela, et c'est probablement aussi parce que l'institution me renvoyait à une faille de garantie de place que j'ai fait appel à toute la ritualisation passée dont j'avais bénéficié pour contrer cette faille.

\section{L'alliance de l'accord minimal et de la séparation psychique}

Il m'a semblé que je pouvais au moins soutenir cela, le droit qu'avait Stéphane de se méfier de moi et de me le signifier. Il se tait là où Mathieu s'embourbait dans des réponses convenues et ce silence, en un sens, me soulage. Je ressens alors que s'installe un accord tacite sur la base de notre désir commun de soutenir une partie du défi et de faire bonne figure vis-à-vis de l'extérieur. La façon dont nous allons le faire nous appartient strictement (en défendant cela, je nous redonne l'espace d'intimité que j'avais laissé échapper avec Mathieu) mais nous allons être capable de le faire. Nous allons jouer le jeu, rester ensemble un certain temps et montrer que "personne n'a mangé personne ». Le reste est une affaire entre nous et ne regarde personne d'autre. Je crois avoir particulièrement ressenti cela à la fin, quand l'éducateur, revenant chercher Stéphane, me demande à nouveau si tout s'est bien passé, et qu'un échange de regards et une esquisse de sourire entre Stéphane et moi nous amène à dire que « oui, oui, tout s'est bien passé ». Aucun de nous deux n'est dupe, chacun de nous sait que l'autre sait, et je déclare, avec son accord, que les autres n'ont pas à savoir.

Dans le même temps, en n'exigeant pas que Stéphane me réponde, tout en signifiant par mes relances que tel est cependant mon désir mais qu'il lui appartient strictement d'y répon- 
dre au non, j'instaure un espace de séparation psychique entre nos deux désirs qui rend la situation raisonnablement sécure. Car face à un Stéphane visiblement peu désireux d'être là, je me pose rapidement la question de lui proposer d'arrêter l'entretien, mais ne m'y résous pas et c'est bien parce que j'ai refusé de substituer son désir au mien, lui signifiant ainsi qu'il devait assumer les conséquences de ses actes (même réticent, il avait malgré tout accepté de venir), que nous avons été obligés de construire un accord sur le respect de nos différences et la nécessité de ne pas «rompre la présence». Le syntagme habituel demanderait plutôt de parler de «rupture du lien », mais il me semble ici que le lien dans son sens ordinaire était refusé, puisque le dialogue ne fonctionnait pas, tandis qu'un « infra-lien » était néanmoins accepté - et peut-être était-ce là l'enjeu de la rencontre - où Stéphane construirait quelque chose de sa capacité d'être seul en présence de l'autre (pour reprendre l'expression de Winnicott). Notons tout de même que cela ne peut se faire sans que sa position ne se fige, le maintenant dans son refus du lien.

La situation n'est donc guère tenable trop longtemps, et au bout du temps que je juge « réglementaire » je mets en question (au sens propre du terme) le désir de Stéphane de s'en aller, l'amenant à me confirmer qu'il n'était pas volontaire pour cet entretien. Ma proposition d'arrêter là entraîne un «Ouais j'veux bien » qui me fait évoquer le terme de « loyal » pour qualifier le moment qui vient de se jouer : sentiment d'un contrat «implicite » où nous avons pu nous respecter mutuellement, même si cela s'est fait au prix d'une obstination de chacun(e) à «tenir » ses enjeux. Si verbalement, peu de chose auront été dites, du moins ai-je eu le sentiment que l'espace instauré permettait à chacun(e) de nous de l'occuper de façon vivante.

\section{Conclusion}

La situation d'entretien clinique de recherche, qui met face à face un(e) interviewé(e) et un(e) chercheur(se) sans autre enjeu que celui da la rencontre elle-même nous permet ainsi de saisir un peu finement ce qui peut se jouer pour ces adolescents considérés comme «en difficulté » et, au-delà, pour 
« nos » adolescent(e)s en général. Les effets de circulation de l'effraction psychique entre les différents protagonistes (chercheuses, jeune, équipe éducative, environnement extérieur à l'institution) peuvent ainsi être interprétés comme une difficulté croissante, dans nos sociétés hypermodernes qui soumettent le sujet à un bombardement sensoriel et émotionnel incessant (voir par exemple Aubert 2003 et Haroche 2008), à situer la limite entre le celui-ci et son environnement. Si bien que l'expression «poser des limites » ne consiste plus seulement, comme on l'entend traditionnellement, à marquer pour le sujet les limites de sa toute-puissance et donc de son emprise sur le monde, mais bien à lui signifier également, en miroir, la nécessité de se soustraire, au moins partiellement, à l'emprise que le monde va prendre sur lui, tout en parvenant à s'y construire une place viable.

Nous voyons en effet comment, face aux sollicitations extrêmement variées dont ils font l'objet, Mathieu comme Stéphane ne parviennent que très difficilement à se situer comme sujet en interaction réelle avec le monde extérieur, puisque le premier se positionne dans l'hyper-soumission et le second dans la résistance obstinée. Ce jeu d'interaction parviendra néanmoins, dans la dynamique de l'écoute clinique, à émerger, de façon extrêmement ténue avec Mathieu et sur un mode un peu plus affirmé avec Stéphane lorsque la différence de nos désirs et la négociation qui en découle pourront se vivre sans trop d'angoisse. Mais, justement parce qu'il s'agit d'une situation de recherche où j'ai pu mettre en place l'espace le plus propice à cette élaboration de la délimitation psychique, nous percevons «en creux » la difficulté qu'il y a à le faire dans des situations ordinaires.

Comment apprendre à vivre si nous ne sommes plus que la résultante des flux sensoriels et émotionnels qui nous affectent ? Comment « devenir quelqu'un » sans la protection rassurante des rituels et des statuts qui présentent ce grand avantage de définir des modes de faire sans demander forcément aux sujets d'y adhérer psychiquement (voir Coppel 1991) ? Je fais l'hypothèse que, dans une société traditionnelle où les rôles sociaux sont strictement définis (au risque, certes, d'entraver les sujets dans leurs modes de faire), le psychisme est relativement protégé dans la mesure où il n'est pas sommé de «coller» à ce qui est mis en scène. À l'opposé, 
dans nos sociétés hypermodernes où chacun doit en permanence se prouver et se justifier, les cadres sociaux traditionnels ne sont plus là pour jouer leur rôle d'étayage psychique et le sujet, privé des " grands récits » fondateurs, éprouverait de plus en plus de difficulté à mettre en place ce que j'ai appelé une "fiction de soi soutenable », c'est-à-dire une histoire de lui-même qui lui permette de vivre (voir Hatchuel 2009).

Piera Aulagnier (1976-1986) nous rappelle l'absolue nécessité, pour qu'une pensée puisse s'élaborer et un «Je » advenir, d'un espace d'intimité psychique à l'abri des intrusions fantasmatiques. Je fais l'hypothèse qu'un rôle social prédéfini peut contribuer à construire un tel espace à l'abri duquel quelque chose du sujet peut prendre le temps de s'élaborer et que sa disparition laisse le champ libre aux logiques de captation de la dette et de mise au défi qui viennent déposséder le sujet de sa capacité de construction d'une fiction de soi. Les adultes éducateurs pourront alors constituer un appui précieux s'ils et elles parviennent à se dégager de leurs propres enjeux psychiques et à créer des espaces où les désirs et les tensions pourront être travaillés, afin d'aider chacun(e) à construire cette fiction de soi et à la soutenir. C'est dans cette optique que nous proposons, à l'université Paris Ouest, un parcours de master de sciences de l'éducation intitulé «formation à l'intervention et à l'analyse de pratiques », centré sur la capacité à mettre en place et animer des espaces élaboratifs, pour les adultes comme pour les jeunes. Ce sont des espaces où nous travaillons à ce que nous appelons une "réintimisation de la pensée », c'està-dire où se construit psychiquement la différence entre intimité partagée et exhibition publique. C'est pourquoi également il me semble que les institutions éducatives doivent porter une attention particulière à ce que $\mathrm{j}$ 'appellerai les « zones frontières », qu'il s'agisse, d'un point de vue géographique, de la délimitation des différents espaces concrets (individuels ou collectifs, privés ou publics) ou d'un point de vue « historique », du travail autour des arrivées et des départs, qui permettent de questionner les liens et les appartenances, c'est-à-dire la façon dont le sujet laisse le monde extérieur le travailler. C'est l'ensemble de ces questions qui m'a conduite à évoquer, cidessus, la «capacité respirante » des institutions et, au-delà, des psychismes qu'elles abritent. 
N. AUBERT (2003), Le culte de l'urgence. La société malade du temps, Paris, Flammarion.

P. Aulagnier (1976), « Le droit au secret : condition pour pouvoir penser », Nouvelle Revue de Psychanalyse, XIV, p. 141-157. Repris dans Un interprète en quête de sens, Paris, Ramsay, 1986, p. 299-324.

C. Blanchard-Laville, P. Chaussecourte, F. Hatchuel, B. Pechberty (2005), «Recherches cliniques d'orientation psychanalytiques dans le champ de l'éducation et de la formation », Revue Française de Pédagogie, $\mathrm{n}^{\circ} 151$, p. 111-162.

M. Coppel (1991), "L'éducateur, le psychanalyste et les mauvaises pensées », Autrement, série «Morales », $\mathrm{n}^{\circ} 2:$ La politesse, vertu des apparences, Paris, Autrement.

G. Devereux (1980), De l'angoisse à la méthode, Paris, Flammarion, (1 1 ère éd. angl. 1967).

C. GefFray (2005), Trésors. Anthropologie analytique de la valeur, Paris, Arcanes.

C. HAROCHE (2008), L'avenir du sensible. Le sens et les sentiments en question, Paris, PUF, coll. «Sociologie d'aujourd'hui ».

F. HATChUEl (2007), Savoir, apprendre, transmettre. Une approche psychanalytique du rapport au savoir, Paris, La Découverte Poche (1 $1^{\mathrm{re}}$ éd. 2005).

F. HATChuel, D. Hans, N. Clerc, V. CARLin (2005), Le rapport au savoir de jeunes en difficultés, rapport final à l'ASEAY (Association pour la Sauvegarde de l'Enfance et de l'Adolescence des Yvelines), Tomes 1 et 2, dact.

F. HATCHUEl (2009), Du rapport au savoir à la fiction de soi : penser, vivre et faire grandir dans un monde incertain. Anthropologie clinique de la transmission. Note de synthèse en vue de l'obtention d'une habilitation à diriger les recherches, Université Paris Ouest Nanterre.

G. Mendel (1993), La société n'est pas une famille, Paris, La Découverte.

P. MerLe (2005), L'élève humilié. L'école : un espace de non droit ?, Paris, PUF.

J.P. PINEL, S. MoRel (2001), «Psychose et institution : défenses paradoxales, anti-processualité et originaire évidé », Revue de psychothérapie psychanalytique de groupe, $\mathrm{n}^{\circ} 36$.

C. Revault D’Allonnes (dir.) (1989), La démarche clinique en sciences humaines, Paris, Dunod.

D.W. WinnicotT (1969), La capacité d'être seul in De la pédiatrie à la psy-

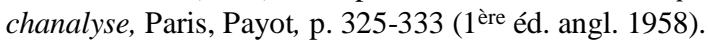

